\title{
Regularizing transformations of polygons
}

\author{
Johann Lang®, Sybille Mick and Otto Röschel
}

\begin{abstract}
We start with a generic $n$-gon $Q_{0}$ with vertices $q_{j, 0}(j=0$, $\ldots, n-1)$ in the $d$-dimensional Euclidean space $\mathbb{E}^{d}$. Additionally, $m+1$ real numbers $u_{0}, \ldots, u_{m} \in \mathbb{R}(m<n)$ with $\sum_{\mu=0}^{m} u_{\mu}=1$ are given. From these initial data we iteratively define generations of $n$-gons $Q_{k}$ in $\mathbb{E}^{d}$ for $k \in \mathbb{N}$ with vertices $q_{j, k}:=\sum_{\mu=0}^{m} u_{\mu} q_{j+\mu, k-1}$. We can show that this affine iteration generally regularizes in an affine sense.
\end{abstract}

Mathematics Subject Classification. 51 N 10, 51 N 20.

Keywords. Affine iterations in higher dimensions, affine regularization, polygons and regularizing iterations, regular $n$-gons.

\section{Introduction}

Schoenberg [6], Ziv [7], Nicollier [2] and Donisi et al. [1] studied geometric iteration processes starting with a generic $n$-gon $Q_{0}$ in $\mathbb{E}^{2}$. They use homotheties to construct vertices of a next generation polygon $Q_{1}$. Reiterating this process creates a series of generations $Q_{k}$. This iteration, in general, has a regularizing effect on the polygon. Surprisingly, the result for $n$-gons in the plane $\mathbb{E}^{2}$ presented by Roeschel in [5] is also valid for $n$-gons in higher dimensions. In [5] the proof for $\mathbb{E}^{2}$ is based on the fact that the space of planar $n$-gons is spanned by the planar prototype $n$-gons of $\mathbb{E}^{2}$. As this does not hold for higher dimensions the proof for $\mathbb{E}^{d}$ with $d \geq 3$ demands another approach with different arguments. We prove an affine regularization theorem: these iterations in higher dimensions also deliver generations $Q_{k}$ approaching the affine shape of regular planar polygons.

\section{The spatial affine iteration}

We use vectors in $\mathbb{R}^{d}$ to describe points of the $d$-dimensional Euclidean space $\mathbb{E}^{d}(d>2)$ with respect to a Cartesian coordinate frame $\left\{O ; x_{1}, \ldots, x_{d}\right\}$. We start with some spatial $n$-gon $Q_{0} \subset \mathbb{E}^{d}$ with vertices $\left\{q_{0,0}, q_{1,0}, \ldots, q_{n-1,0}\right\}$ 

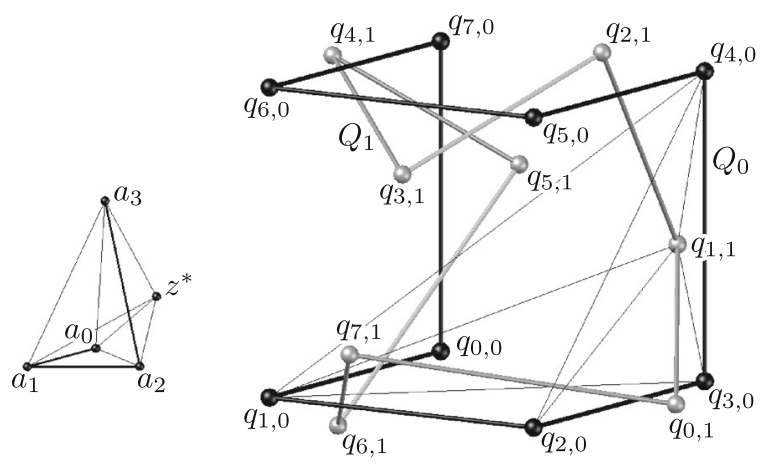

FiguRE 1 An example for $n=8$ and $m=d=3$ : the polygon $Q_{0}$ with vertices of a cube and the first generation polygon $Q_{1}$ for $\left(u_{0}, u_{1}, u_{2}, u_{3}\right)=(0.2,-0.35,0.75,0.4)$

$\left(n>2, q_{j, 0} \in \mathbb{R}^{d}\right)$. Our starting polygon $Q_{0}$ shall be called polygon of generation 0 .

On the other hand in an $m$-dimensional affine space $\mathbb{R}^{m}(0<m<n)$ with a simplex $S:=\left\{a_{0}, \ldots, a_{m}\right\}$ we choose a reference point $z^{*}$ with respect to $S$ : Let $z^{*}:=\sum_{\mu=0}^{m} u_{\mu} a_{\mu}$ be given by its barycentric coordinates $\left(u_{0}, \ldots, u_{m}\right) \in$ $\mathbb{R}^{1 \times(m+1)}$ with $\sum_{\mu=0}^{m} u_{\mu}=1$.

Let $\alpha_{j, 1}$ be the affine mappings from the ordered reference simplex vertex set $S$ to ordered sets of $m$ consecutive vertices $q_{j, 0}, \ldots, q_{j+m, 0}$ of $Q_{0}(j \in \mathbb{J}:=$ $\{0, \ldots, n-1\}$; first index $\bmod n)$. Each of these $n$ affine mappings is applied to the reference point $z^{*}$; this way we get $n$ image points $q_{j, 1}:=\alpha_{j, 1}\left(z^{*}\right)=$ $\sum_{\mu=1}^{m} u_{\mu} q_{j+\mu-1,0}$ which form a new $n$-gon $Q_{1}$ called the generation 1 polygon.

The same process can now be applied, in turn, to the polygon $Q_{1}$ with the same reference simplex $S$ and the same reference point $z^{*}$, creating a subsequent polygon $Q_{2}$. Iteration yields a series of polygons. $Q_{k}:=\left\{q_{0, k}, \ldots, q_{n-1, k}\right\}$ is the $k$ th generation polygon with vertices

$$
q_{j, k}=\sum_{\mu=0}^{m} u_{\mu} q_{j+\mu, k-1} \in \mathbb{R}^{d} \quad(j \in \mathbb{J}, k \in \mathbb{N} \backslash\{0\}) .
$$

The procedure is a $d$-dimensional generalisation of the geometric iteration presented in [5]. Figure 1 shows the first iteration step for an example with $n=8$ and $m=d=3$.

\section{The iteration process}

We describe the polygons $Q_{k}$ by $d \times n$-matrices $Q_{k}:=\left(q_{0, k}, \ldots, q_{n-1, k}\right)$ in $\mathbb{R}^{d \times n}$ with $q_{j, k}(2.1)$. Formula (2.1) can be rewritten as a product of matrices $Q_{k}:=Q_{k-1} \cdot M$ with the circulant $n \times n$-matrix $M \in \mathbb{R}^{n \times n}$ : 


$$
M=\left(\begin{array}{cccccccccc}
u_{0} & 0 & \cdots & \cdots & 0 & u_{m} & u_{m-1} & \cdots & u_{2} & u_{1} \\
u_{1} & \ddots & \ddots & & & \ddots & \ddots & \ddots & & u_{2} \\
\vdots & \ddots & \ddots & \ddots & & & \ddots & \ddots & \ddots & \vdots \\
\vdots & & \ddots & \ddots & \ddots & & & \ddots & \ddots & u_{m-1} \\
u_{m-1} & & & \ddots & \ddots & \ddots & & & \ddots & u_{m} \\
u_{m} & \ddots & & & \ddots & \ddots & \ddots & & & 0 \\
0 & \ddots & \ddots & & & \ddots & \ddots & \ddots & & \vdots \\
\vdots & \ddots & \ddots & \ddots & & & \ddots & \ddots & \ddots & \vdots \\
\vdots & & \ddots & \ddots & \ddots & & & \ddots & \ddots & 0 \\
0 & \cdots & \cdots & 0 & u_{m} & u_{m-1} & \cdots & \cdots & u_{1} & u_{0}
\end{array}\right)
$$

The $n$th complex roots of unity $\in \mathbb{C}$ shall be termed $\zeta_{j}:=\exp \left(i \frac{2 j \pi}{n}\right)=$ $\cos \frac{2 j \pi}{n}+i \sin \frac{2 j \pi}{n}(j \in \mathbb{Z})$. We define the vectors

$$
P_{j}:=\left(\zeta_{j}^{0}, \ldots, \zeta_{j}^{n-1}\right) \in \mathbb{C}^{1 \times n} \quad(j \in \mathbb{Z})
$$

and have $P_{j} \cdot M=P_{j} \sum_{\mu=0}^{m} u_{\mu} \zeta_{j}^{\mu}$ and $M \cdot P_{n-j}^{t}=\left(\sum_{\mu=0}^{m} u_{\mu} \zeta_{j}^{\mu}\right) P_{n-j}^{t}$. Thus, the vectors $P_{j}$ and $P_{n-j}^{t}(j \in \mathbb{J})$ are left and right eigenvectors of $M$. The corresponding eigenvalue is

$$
\lambda_{j}:=\sum_{\mu=0}^{m} u_{\mu} \zeta_{j}^{\mu}(j \in \mathbb{J}) .
$$

As $\left(u_{0}, \ldots, u_{m}\right) \in \mathbb{R}^{1 \times(m+1)}$ and $\overline{\zeta_{j}^{\mu}}=\zeta_{n-j}^{\mu}$ we have $\bar{\lambda}_{j}=\lambda_{n-j}$ for all $j \in$ $\mathbb{J} \backslash\{0\}$.

We now regard two matrices out of $\mathbb{C}^{n \times n}$

$$
L:=\frac{1}{\sqrt{n}}\left(\begin{array}{c}
P_{0} \\
\vdots \\
P_{n-1}
\end{array}\right) \text { and } R:=\frac{1}{\sqrt{n}}\left(\begin{array}{c}
P_{n} \\
\vdots \\
P_{1}
\end{array}\right) \text {. }
$$

$L$ and $R$ are symmetric and regular for $n>1$ (see [3,5,6] and [7]). We have: $L=\bar{R}$ and $L \cdot R=I_{n, n}$ with the $n \times n$ - unit matrix $I_{n, n}$; the matrices $L$ and $R$ are unitary $n \times n$-matrices in $\mathbb{C}^{n \times n}$. We have $L \cdot M \cdot R=D\left(\lambda_{0}, \ldots, \lambda_{n-1}\right)$ with the diagonal matrix $D\left(\lambda_{0}, \ldots, \lambda_{n-1}\right) \in \mathbb{C}^{n \times n}$ containing the eigenvalues $\lambda_{j}$ of $M$ as its elements in the main diagonal. This yields $M=R \cdot D\left(\lambda_{0}, \ldots, \lambda_{n-1}\right) \cdot L$ and

$$
\begin{aligned}
& Q_{k} \cdot R=Q_{k-1} \cdot R \cdot D\left(\lambda_{0}, \ldots, \lambda_{n-1}\right) \text { and } \\
& Q_{k} \cdot R=Q_{0} \cdot R \cdot D\left(\lambda_{0}, \ldots, \lambda_{n-1}\right)^{k} \quad \text { for } k \in \mathbb{N} \backslash\{0\} .
\end{aligned}
$$

We get $Q_{k} \cdot R=\frac{1}{\sqrt{n}}\left(\sum_{\nu=0}^{n-1} q_{\nu, k} \zeta_{n}^{\nu}, \sum_{\nu=0}^{n-1} q_{\nu, k} \zeta_{n-1}^{\nu}, \ldots, \sum_{\nu=0}^{n-1} q_{\nu, k} \zeta_{1}^{\nu}\right)$.

Then (3.5) yields

$$
\sum_{\nu=0}^{n-1} q_{\nu, k} \zeta_{n-j}^{\nu}=\lambda_{j}^{k} \sum_{\nu=0}^{n-1} q_{\nu, 0} \zeta_{n-j}^{\nu} \quad \forall j \in \mathbb{J} .
$$


Due to $\lambda_{0}=\sum_{\mu=0}^{m} u_{\mu} \zeta_{0}^{\mu}=1$ and $\zeta_{n}^{\nu}=1$, the index $j=0$ in (3.6) delivers $\sum_{\nu=0}^{n-1} q_{\nu, k}=\sum_{\nu=0}^{n-1} q_{\nu, 0}$ for all $k \in \mathbb{N} \backslash\{0\}$ : All polygons $Q_{k}$ have the same center of gravity.

From now on let the initial polygon $Q_{0}$ have its center of gravity in the origin $O:=(0, \ldots, 0)^{t}$. So we can be sure that for all $k \in \mathbb{N}$

$$
\frac{1}{n} \sum_{\nu=0}^{n-1} q_{\nu, k}=\mathbf{o}_{d}:=(0, \ldots, 0)^{t} .
$$

As the matrix $R$ is regular the initial polygon $Q_{0}$ can explicitly be retrieved from the $d \times n$ - matrix

$$
Q_{0} \cdot R=: B=\left(b_{0}, \ldots, b_{n-1}\right) \in \mathbb{C}^{d \times n} \quad \text { with } \quad b_{j}=\frac{1}{\sqrt{n}} \sum_{\nu=0}^{n-1} q_{\nu, 0} \zeta_{n-j}^{\nu} \in \mathbb{C}^{d} .
$$

From $q_{\nu, 0} \in \mathbb{R}^{d}$ and $\zeta_{n-j}^{\nu}=\overline{\zeta_{j}^{\nu}}$ we get $\overline{b_{j}}=b_{n-j}$ for all $j \in \mathbb{J}^{*}:=\{1, \ldots, n-1\}$. Because of (3.7) the first column vector is zero: $b_{0}=\mathbf{o}_{d}$. Equation (3.5) yields

$$
Q_{k} \cdot R=B \cdot D\left(\lambda_{0}, \ldots, \lambda_{n-1}\right)^{k}=\left(\mathbf{o}_{d}, \lambda_{1}^{k} b_{1}, \ldots, \lambda_{n-1}^{k} b_{n-1}\right) .
$$

Thus, we do not alter the recursion in any way if we replace the diagonal matrix $D\left(\lambda_{0}, \ldots, \lambda_{n-1}\right)$ in $(3.5)$ by the diagonal matrix $D^{*}:=D\left(0, \lambda_{1}, \ldots, \lambda_{n-1}\right)$. With this in mind, the iteration process can be described by

$$
Q_{k}=B \cdot D^{* k} \cdot L=\frac{1}{\sqrt{n}} \sum_{\nu=1}^{n} \lambda_{\nu}^{k} b_{\nu} P_{\nu} \Leftrightarrow q_{j, k}=\frac{1}{\sqrt{n}} \sum_{\nu=1}^{n} \lambda_{\nu}^{k} b_{\nu} \zeta_{j}^{\nu}
$$

for $j \in \mathbb{J}$. Note that $b_{\nu} P_{\nu} \in \mathbb{C}^{d \times n}$ for $\nu \in \mathbb{J}^{*}$.

\section{Prototype polygons}

The Gaussian plane of complex numbers $\mathbb{C}$ can be interpreted as a Euclidean plane $\mathbb{E}^{2}$ with a Cartesian coordinate frame $\{O ; 1, i\}$. We embed $\mathbb{E}^{2}$ into $\mathbb{E}^{d}$ by identifying 1 and $i$ with the $d$-dimensional unit vectors $e_{1}:=(1,0,0, \ldots, 0)^{t}$ and $e_{2}:=(0,1,0, \ldots, 0)^{t}$, respectively. The elements of $P_{j}(3.2)$ can be viewed as a collection of $n$ points $\zeta_{j}^{\nu}(\nu \in \mathbb{J})$ equally distributed on the unit circle of $\mathbb{E}^{2} \subset \mathbb{E}^{d}$ centered in $O$ with $j \in \mathbb{J}^{*}:=\{1, \ldots, n-1\}$. Its points can be written as

$$
T_{j}=e_{1} \frac{P_{j}+\bar{P}_{j}}{2}+e_{2} \frac{P_{j}-\bar{P}_{j}}{2 i}=e_{1} \frac{P_{j}+P_{n-j}}{2}+e_{2} \frac{P_{j}-P_{n-j}}{2 i} .
$$

$T_{j}$ is represented by a matrix $\in \mathbb{R}^{d \times n}$ with columns

$$
t_{\nu, j}:=\left(\cos \frac{2 \pi \nu j}{n}, \sin \frac{2 \pi \nu j}{n}, 0, \ldots, 0\right)^{t} \in \mathbb{R}^{d}(\nu \in \mathbb{J}) .
$$

$T_{j}$ forms the so-called 'regular prototype $n$-gon of jth kind'. The regular $n$-gon $T_{n-j}$ is symmetric to $T_{j}$ w.r.t. the axis $e_{1}$ and thus affinely equivalent to $T_{j}$. If $j$ and $n$ are relatively prime the polygon $T_{j}$ is either a regular $n$-gon or an 
$n$-sided regular star. If $j$ is a divisor of $n$ with $n=j p$ the polygon $T_{j}$ is either a regular $p$-gon or an ordinary regular star with $p$ vertices, each of the vertices being multiply counted ( $j$ times).

\section{The concept of affine regularization}

An affine mapping of $\mathbb{E}^{d}$ keeping the origin $O$ in its place is described by

$$
\beta: \mathbb{E}^{d} \longrightarrow \mathbb{E}^{d}, x \mapsto \beta(x)=C x \text { with } C \in \mathbb{R}^{d \times d} .
$$

The affine image of the polygon $Q_{k}=\left(q_{0, k}, \ldots, q_{n-1, k}\right)$ is $\beta\left(Q_{k}\right):=C \cdot Q_{k}$. Our iteration $(2.1)$ seems to regularize for certain $\left(u_{0}, \ldots, u_{m}\right) \in \mathbb{R}^{1 \times(m+1)}$ irrespective of the choice of the starting polygon $Q_{0}$. In order to examine this interesting peculiarity we compare the $n$-gons $Q_{k}$ with a regular prototype $n$-gon $T_{j}(4.1)$ of $j$ th kind $^{1}$ :

Definition 5.1. We call the iteration (2.1) affinely regularizing of kind $j$ with $1 \leq j \leq n / 2$ if, for any generic initial polygon $Q_{0}$, there exist affine mappings $\beta_{k}: \mathbb{E}^{d} \longrightarrow \mathbb{E}^{d}$ transforming $Q_{k}=\left(q_{0, k}, \ldots, q_{n-1, k}\right)^{t}$ into polygons $\beta_{k}\left(Q_{k}\right)$ with the property that the series $\Delta_{k}$ of sums of the squared distances

$$
\Delta_{k}:=\sum_{\nu=0}^{n-1}\left\|\beta_{k}\left(q_{\nu, k}\right)-t_{\nu, j}\right\|^{2}=\operatorname{tr}\left(\left(T_{j}-\beta_{k}\left(Q_{k}\right)\right)^{t} \cdot\left(\overline{T_{j}-\beta_{k}\left(Q_{k}\right)}\right)\right)
$$

of respective vertices of $\beta\left(Q_{k}\right)$ and of the regular prototype polygon $T_{j}$ of $j$ th kind is a null series: $\lim _{k \rightarrow \infty} \Delta_{k}=0$.

\section{The affine regularization theorem}

The shape of the polygons $Q_{k}$ depends on the input data set $Q_{0}$ and on the barycentric coordinates $\left(u_{0}, \ldots, u_{m}\right)$ of the reference point $z^{*}$ with $\sum_{\mu=0}^{m} u_{\mu}=$ 1. The latter determine the matrix $M(3.1)$, the eigenvalues $\lambda_{j}$ and the diagonal matrix $D^{*}=D\left(0, \lambda_{1}, \ldots, \lambda_{n-1}\right)$. The norms $n_{j}:=\left|\lambda_{j}\right|$ of $\lambda_{j}$ for $j \in \mathbb{J}^{*}$ are given by

$$
n_{j}^{2}=\lambda_{j} \overline{\lambda_{j}}=\sum_{\mu, \nu=0}^{m} u_{\mu} u_{\nu} \zeta_{j}^{\mu-\nu}
$$

We put $N:=\max \left\{n_{1}, \ldots, n_{n-1}\right\}$. Let the barycentrics $\left(u_{0}, \ldots, u_{m}\right)$ be chosen generally such that not all $\lambda_{1}, \ldots, \lambda_{n-1}$ vanish. $N=0$ is equivalent with $\lambda_{1}=\cdots=\lambda_{n-1}=0$ and can only occur if $m=n-1$ and, additionally, $\left(u_{0}, \ldots, u_{n-1}\right)=(1 / n, \ldots, 1 / n)$. This case of iterated series of 'degenerate $n$ gons' $Q_{k}$, all collapsing into the center of gravity $O$ shall be excluded further on. For $0<N<1$ the series $Q_{k}$ gradually contracts for increasing $k$ and tends towards the center of gravity $O$. For $N=1$ the series $Q_{k}$ remains finite,

\footnotetext{
${ }^{1}$ As the prototypes $T_{j}$ and $T_{n-j}$ are affinely equivalent, an iteration regularizing of $j$ th kind will also be regularizing of kind $n-j$ and we can confine ourselves to $1 \leq j \leq n / 2$.
} 
but in general still may change its shape and its position from generation to generation. For $N>1$ the series $Q_{k}$ gradually expands for increasing $k$.

We will prove that for any $N>0$, the algorithm is - in general-affinely regularizing. We divide the set of indices into two distinct subsets:

$$
\mathbb{J}_{1}:=\left\{j \in \mathbb{J}^{*} /\left|\lambda_{j}\right|=N\right\} \neq \emptyset \quad \text { and } \quad \mathbb{J}_{2}:=\mathbb{J}^{*} \backslash \mathbb{J}_{1} .
$$

According to $(3.3)$, for any $j^{*} \in \mathbb{J}_{1}$ the index $n-j^{*}$ is also contained in $\mathbb{J}_{1}$; for even $n$ and $j^{*}=n / 2$ these two indices coincide. We have

$$
\frac{\left|\lambda_{j}\right|}{N}=1 \forall j \in \mathbb{J}_{1} \quad \text { and } \quad 0 \leq \frac{\left|\lambda_{j}\right|}{N}<1 \forall j \in \mathbb{J}_{2} .
$$

Equations (3.10) yield

$$
\begin{gathered}
Q_{k}=\frac{N^{k}}{\sqrt{n}}\left(\sum_{\nu \in \mathbb{J}_{1}}\left(\frac{\lambda_{\nu}}{N}\right)^{k} b_{\nu} P_{\nu}+\sum_{\nu \in \mathbb{J}_{2}}\left(\frac{\lambda_{\nu}}{N}\right)^{k} b_{\nu} P_{\nu}\right) \\
\Leftrightarrow q_{j, k}=\frac{N^{k}}{\sqrt{n}}\left(\sum_{\nu \in \mathbb{J}_{1}}\left(\frac{\lambda_{\nu}}{N}\right)^{k} b_{\nu} \zeta_{\nu}^{j}+\sum_{\nu \in \mathbb{J}_{2}}\left(\frac{\lambda_{\nu}}{N}\right)^{k} b_{\nu} \zeta_{\nu}^{j}\right) .
\end{gathered}
$$

Regardless of the input data $b_{j}(3.8)$ the coefficients $\left(\frac{\lambda_{\nu}}{N}\right)^{k}$ form null series for all $\nu \in \mathbb{J}_{2}$ and $k \rightarrow \infty$; the coefficients $\left(\frac{\lambda_{\nu}}{N}\right)^{k}$ for all $\nu \in \mathbb{J}_{1}$ are complex numbers of norm 1 for all $k \in \mathbb{N}$.

$Q_{k}$ and any homothetic image $\rho_{k}\left(Q_{k}\right)$ have the same affine shape. Following Definition 5.1 we can apply homotheties $\rho_{k}: \mathbb{E}^{d} \longrightarrow \mathbb{E}^{d}$ with $x \mapsto x \frac{\sqrt{n}}{N^{k}}$. These homotheties $\rho_{k}$ turn (6.4) into

$$
\begin{gathered}
\rho_{k}\left(Q_{k}\right)=\sum_{\nu \in \mathbb{J}_{1}}\left(\frac{\lambda_{\nu}}{N}\right)^{k} b_{\nu} P_{\nu}+\sum_{\nu \in \mathbb{J}_{2}}\left(\frac{\lambda_{\nu}}{N}\right)^{k} b_{\nu} P_{\nu} \\
\Leftrightarrow \rho_{k}\left(q_{j, k}\right)=\sum_{\nu \in \mathbb{J}_{1}}\left(\frac{\lambda_{\nu}}{N}\right)^{k} b_{\nu} \zeta_{\nu}^{j}+\sum_{\nu \in \mathbb{J}_{2}}\left(\frac{\lambda_{\nu}}{N}\right)^{k} b_{\nu} \zeta_{\nu}^{j} .
\end{gathered}
$$

With reference to the cardinal number of the index set $\mathbb{J}_{1}$ we have three cases:

Case A: The index set $\mathbb{J}_{1}$ contains just one element. This can only happen if $n$ is an even integer and the barycentrics $\left(u_{0}, \ldots, u_{m}\right)$ lead to $\mathbb{J}_{1}=\{n / 2\}$. We have $\zeta_{n / 2}=-1$, and $\lambda_{n / 2}=\sum_{\mu=0}^{m} u_{\mu}(-1)^{\mu} \in \mathbb{R}$. As $N=\left|\lambda_{n / 2}\right|>0$ and therefore $\lambda_{n / 2}= \pm N \neq 0$ formula (6.5) reads as

$$
\rho_{k}\left(Q_{k}\right)=( \pm 1)^{k} b_{n / 2} P_{n / 2}+\sum_{\nu \in \mathbb{J}_{2}}\left(\frac{\lambda_{\nu}}{N}\right)^{k} b_{\nu} P_{\nu}
$$

For every $k$ we apply a further homothety $\sigma_{k}: \mathbb{E}^{d} \longrightarrow \mathbb{E}^{d}$ with

$$
\sigma_{k}(x)=( \pm 1)^{k} x \Rightarrow \sigma_{k}\left(\rho_{k}\left(Q_{k}\right)\right)=b_{n / 2} P_{n / 2}+\sum_{\nu \in \mathbb{J}_{2}}\left(\frac{ \pm \lambda_{\nu}}{N}\right)^{k} b_{\nu} P_{\nu}
$$

We have $b_{n / 2}=\sum_{\nu=0}^{n-1} q_{\nu, 0} \zeta_{n / 2}^{\nu}=\sum_{\nu=0}^{n-1}(-1)^{\nu} q_{\nu, 0} \in \mathbb{R}^{d}$. For a generic input polygon $Q_{0}$ we can assume $b_{n / 2} \neq \mathbf{o}_{d}$. In this case we choose an affine mapping $\tau$ with fixed point $O$ and $b_{n / 2} \mapsto e_{1} \in \mathbb{R}^{d}$. The mapping $\tau$ induces an affine mapping $\mathbb{C}^{d} \longrightarrow \mathbb{C}^{d}$ transforming $b_{\nu}\left(\nu \in \mathbb{J}_{2}\right)$ into $b_{\nu}^{*}:=\tau\left(b_{\nu}\right) \in \mathbb{C}^{d}$; the 
vectors $b_{\nu}^{*}$ do not depend on $k$. The affine mapping $\beta_{k}:=\tau \circ \sigma_{k} \circ \rho_{k}$ places the $k$ th generation polygon $Q_{k}$ into

$$
\beta_{k}\left(Q_{k}\right)=e_{1} P_{n / 2}+\sum_{\nu \in \mathbb{J}_{2}}\left(\frac{ \pm \lambda_{\nu}}{N}\right)^{k} b_{\nu}^{*} P_{\nu} .
$$

The distance vectors $d_{j, k}$ of the vertices of $\beta_{k}\left(Q_{k}\right)$ to the respective vertices of the prototype polygon $T_{n / 2}=e_{1} P_{n / 2}$ (4.1) are the columns of $D_{k}=\left(d_{0, k}, \ldots, d_{n-1, k}\right)$ with

$$
D_{k}=\sum_{\nu \in \mathbb{J}_{2}}\left(\frac{ \pm \lambda_{\nu}}{N}\right)^{k} b_{\nu}^{*} P_{\nu} \Leftrightarrow d_{j, k}=\sum_{\nu \in \mathbb{J}_{2}}\left(\frac{ \pm \lambda_{\nu}}{N}\right)^{k} b_{\nu}^{*} \zeta_{\nu}^{j} \quad(j \in \mathbb{J}) .
$$

The vectors $b_{\nu}^{*} \zeta_{\nu}^{j}$ are independent from $k$. As the norms of $\left(\frac{\lambda_{\nu}}{N}\right)^{k}$ form null series for all $\nu \in \mathbb{J}_{2}$ we can be sure that $\lim _{k \rightarrow \infty} d_{j, k}=\mathbf{o}_{d}$ for all $j \in \mathbb{J}$. The sum of the squared distances $\Delta_{k}:=\sum_{j=0}^{n-1}\left\|d_{j, k}\right\|^{2}$ is a null series: $\lim _{k \rightarrow \infty} \Delta_{k}=0$. Thus, according to our Definition 5.1 the iteration process in case A is affinely regularizing of kind $n / 2$. For generic input $Q_{0}$ the polygons $Q_{k}$ approach the shape of the $n$-gon $T_{n / 2}$. The straight lines approximating the polygons $Q_{k}$ tend towards the straight line through $O$ with direction vector $b_{n / 2}$.

Case B: The index set $\mathbb{J}_{1}$ contains exactly two different elements: $\mathbb{J}_{1}=\left\{j^{*}, n-\right.$ $\left.j^{*}\right\}$ with $1 \leq j^{*}<n / 2$. In a way, this could be considered the general case. We put $\lambda_{j^{*}}=N e^{i \phi}$ and $\lambda_{n-j^{*}}=N e^{-i \phi}$ with some real angle $\phi \in[0,2 \pi)$ and define $W:=\sum_{\nu \in \mathbb{J}_{2}}\left(\frac{\lambda_{\nu}}{N}\right)^{k} b_{\nu} P_{\nu}$. Then (6.5) yields

$$
\rho_{k}\left(Q_{k}\right)=e^{i k \phi} b_{j^{*}} P_{j^{*}}+e^{-i k \phi} b_{n-j^{*}} \bar{P}_{j^{*}}+W .
$$

Let $b_{j^{*}}:=x+i y$ with $x, y \in \mathbb{R}^{d}$. We then have $b_{n-j^{*}}=\bar{b}_{j^{*}}=x-i y$ and

$$
\rho_{k}\left(Q_{k}\right)=x\left(e^{i k \phi} P_{j^{*}}+e^{-i k \phi} \bar{P}_{j^{*}}\right)+i y\left(e^{i k \phi} P_{j^{*}}-e^{-i k \phi} \bar{P}_{j^{*}}\right)+W .
$$

For a generic input $n$-gon $Q_{0}$ the two vectors $x, y \in \mathbb{R}^{d}$ are linearly independent. Let $\sigma: \mathbb{E}^{d} \longrightarrow \mathbb{E}^{d}$ be any affine mapping that maps the two vectors $x, y$ into $\sigma(x):=e_{1} / 2$ and $\sigma(y):=-e_{2} / 2 . \sigma$ induces an affine mapping $\mathbb{C}^{d} \longrightarrow \mathbb{C}^{d}$ transforming $b_{\nu}\left(\nu \in \mathbb{J}_{2}\right)$ into $\sigma\left(b_{\nu}\right)$. We have

$$
\begin{aligned}
\sigma\left(\rho_{k}\left(Q_{k}\right)\right)= & \left(e_{1} \cos k \phi+e_{2} \sin k \phi\right) \frac{P_{j^{*}}+\bar{P}_{j^{*}}}{2} \\
& +\left(-e_{1} \sin k \phi+e_{2} \cos k \phi\right) \frac{P_{j^{*}}-\bar{P}_{j^{*}}}{2 i} \\
& +\sum_{\nu \in \mathbb{J}_{2}}\left(\frac{\lambda_{\nu}}{N}\right)^{k} \sigma\left(b_{\nu}\right) P_{\nu} .
\end{aligned}
$$

We define the complex numbers $\theta_{\mu, \nu}:=\sigma\left(b_{\mu}\right)^{t} \overline{\sigma\left(b_{\nu}\right)}$ for $\mu, \nu \in \mathbb{J}_{2}$. The matrices

$$
R_{k}:=\left(\begin{array}{ccccc}
\cos k \phi & \sin k \phi & 0 & \ldots & 0 \\
-\sin k \phi & \cos k \phi & 0 & \ldots & 0 \\
0 & 0 & 1 & \ldots & 0 \\
\vdots & \vdots & \vdots & \ddots & \vdots \\
0 & 0 & 0 & \ldots & 1
\end{array}\right)
$$


describe rotations $\tau_{k}$ in $\mathbb{E}^{d}$. The induced mappings $\tau_{k}$ in $\mathbb{C}^{d}$ transform the vectors $\sigma\left(b_{\nu}\right)$ into vectors $\tau_{k}\left(\sigma\left(b_{\nu}\right)\right) \in \mathbb{C}^{d}\left(\nu \in \mathbb{J}_{2}\right)$. The mappings $\beta_{k}:=\tau_{k} \circ$ $\sigma \circ \rho_{k}$ are affine mappings from $\mathbb{E}^{d}$ into $\mathbb{E}^{d}$ and deliver

$$
\beta_{k}\left(Q_{k}\right)=e_{1} \frac{P_{j^{*}}+\bar{P}_{j^{*}}}{2}+e_{2} \frac{P_{j^{*}}-\bar{P}_{j^{*}}}{2 i}+\sum_{\nu \in J_{2}}\left(\frac{\lambda_{\nu}}{N}\right)^{k} \tau_{k}\left(\sigma\left(b_{\nu}\right)\right) P_{\nu} .
$$

As every $\tau_{k}$ preserves scalar products we have $\tau_{k}\left(\sigma\left(b_{\mu}\right)\right)^{t} \overline{\tau_{k}\left(\sigma\left(b_{\nu}\right)\right)}=\theta_{\mu, \nu}$ for all $\mu, \nu \in \mathbb{J}_{2}$. According to (5.2) we compute the sum of squared distances of the vertices of $\beta_{k}\left(Q_{k}\right)$ to the respective vertices of the prototype polygon $T_{j *}$ and arrive at

$$
\Delta_{k}=\operatorname{tr}\left(\left(T_{j^{*}}-\beta_{k}\left(Q_{k}\right)\right)^{t} \cdot\left(\overline{T_{j^{*}}-\beta_{k}\left(Q_{k}\right)}\right)\right)=n \sum_{\mu \in \mathbb{J}_{2}}\left(\frac{\lambda_{\mu} \bar{\lambda}_{\mu}}{N^{2}}\right)^{k} \theta_{\mu, n-\mu}
$$

As the values $\theta_{\mu, n-\mu}$ are independent from $k$ and $0 \leq \frac{\lambda_{\mu} \bar{\lambda}_{\mu}}{N^{2}}<1$ for all $\mu \in \mathbb{J}_{2}$ the values $\Delta_{k}(k \in \mathbb{N})$ form a null series. Accordingly, the corresponding iteration process in case B is regularizing of kind $j^{*}$ with $1 \leq j^{*}<n / 2$. For generic input $n$-gons $Q_{0}$ the two vectors $x$ and $y$ determine a plane $\varepsilon^{*}$ through $O$. The planes $\varepsilon_{k}$ approximating the polygon $Q_{k}$ tend towards $\varepsilon^{*}$.

Case $\mathbf{C}$ : The index set $\mathbb{J}_{1}$ contains more than two different elements. We have $j^{*}, j^{* *}, n-j^{*} \in \mathbb{J}_{1}$ with $1 \leq j^{*}<j^{* *} \leq n / 2$. According to (6.1) this is characterized by

$$
\sum_{\mu, \nu=0}^{m} u_{\mu} u_{\nu} \zeta_{j^{* *}}^{\mu-\nu}=\sum_{\mu, \nu=0}^{m} u_{\mu} u_{\nu} \zeta_{j^{*}}^{\mu-\nu}
$$

The coefficients of $u_{\mu} u_{\nu}$ in (6.16) are $\zeta_{j^{* *}}^{\mu-\nu}+\zeta_{j^{* *}}^{\nu-\mu}-\zeta_{j^{*}}^{\mu-\nu}-\zeta_{j^{*}}^{\nu-\mu} \in \mathbb{R}$. The corresponding barycentrics $\left(u_{0}, \ldots, u_{m}\right)$ denote points $z^{*} \in \mathbb{R}^{m}$ which, in general, are positioned on an $(m-1)$-dimensional quadric of $\mathbb{R}^{m}$ containing the vertices of the simplex $S$. In this case we cannot prove any regularizing effect of the affine iteration.

We call the barycentrics $\left(u_{0}, \ldots, u_{m}\right)$ 'generic' if they do not lead to Case $\mathrm{C}$ or, for $m=n-1$, they are different from $\left(\frac{1}{n}, \ldots, \frac{1}{n}\right)$. Overall, we have

Theorem 6.1. Affine Regularization Theorem. For generic barycentrics the iteration process (2.1) is affinely regularizing according to Definition 5.1. The barycentrics $\left(u_{0}, \ldots, u_{m}\right)(m<n)$ determine the eigenvalues $\lambda_{1}, \ldots, \lambda_{n-1}$ given by (3.3) and their maximal norm $N>0$. If there is exactly one index $j^{*}$ (with $1 \leq j^{*} \leq n / 2$ ) with eigenvalue $\lambda_{j^{*}}$ of norm $N$ the iteration is regularizing of kind $j^{*}$.

If the iteration is affinely regularizing of kind $j^{*}$ then, for a generic input $n$-gon $Q_{0}$, the shape of $Q_{k}$ gradually approaches the shape of an affinely transformed prototype $n$-gon $T_{j^{*}}$. 

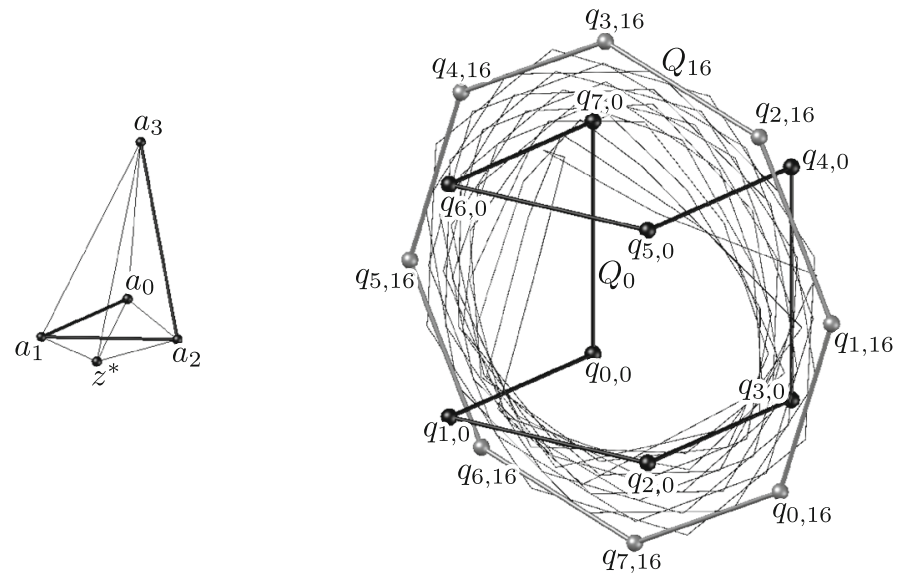

FiguRE 2 An example with $n=8, m=d=3$ with $\mathbb{J}_{1}=\{1,7\}$

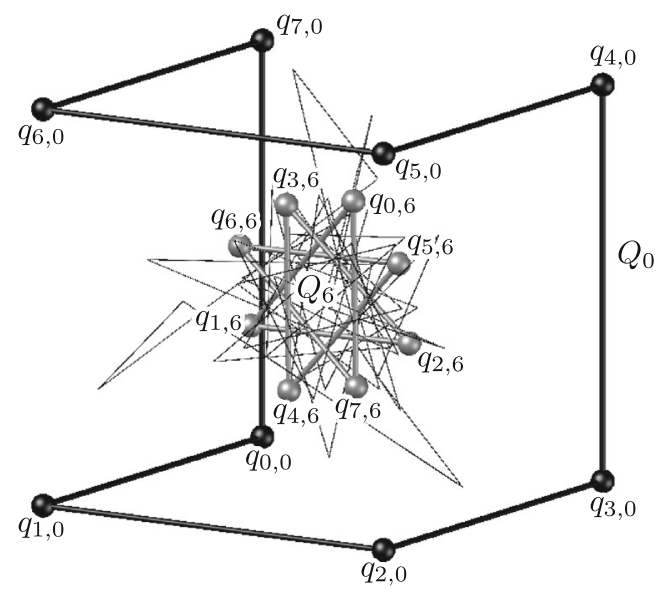

Figure 3 An exceptional example for $n=8, m=d=3$ with $b_{4}=\mathbf{o}_{d}$ and $\mathbb{J}_{1}=\{4\}$. For this specific $n$-gon $Q_{0}$ the algorithm works as if it was affinely regularizing of kind 3

Figure 2 shows an example for the same initial octogon $Q_{0}$ as in Fig. 1 $(n=8, m=d=3)$. Here the barycentrics of $z^{*}$ are $\left(u_{0}, u_{1}, u_{2}, u_{3}\right)=$ $(0.4,0.5,0.3,-0.2)$. We get $n_{1} \approx 1.03, n_{2} \approx 0.71, n_{3} \approx 0.13, n_{4} \approx 0.4$ and therefore we have $\mathbb{J}_{1}=\{1,7\}$. The algorithm is regularizing of kind 1 (case B). The figure shows $Q_{0}$ and the following generations up to $Q_{16}$.

\section{Remarkable exceptions}

For specific initial polygons $Q_{0}$ the algorithm may deliver unexpected results. If the coefficient vectors $b_{\nu}$ of the regarded eigenvalues $\lambda_{\nu}$ for $\nu \in \mathbb{J}_{1}$ in (6.4) 
vanish the respective eigenvalues have no influence on the regularizing process. So, for such a specific $n$-gon $Q_{0}$, the algorithm works in the same way as if in (3.10) these eigenvalues $\lambda_{\nu}$ had been replaced by $\lambda_{\nu}=0$. The remaining eigenvalues deliver another maximum norm $N^{*}<N$ and a different set $\mathbb{J}_{1}$. Now our classification (Sect. 6) reveals the affine shape of the series $Q_{k}$.

Figure 3 shows such an example for $n=8, m=d=3$ where we have $\left(u_{0}, u_{1}, u_{2}, u_{3}\right)=(0.5,-0.25,0.5,0.25)$. We get $n_{1} \approx 0.52, n_{2}=0.5, n_{3} \approx$ $0.99<1, n_{4}=1$. Hence $N=1$ and we conclude that the algorithm is affinely regularizing of kind $4 ; Q_{k}$ is expected to approach the shape of the prototype is $T_{4}$ which is a line segment. The special initial octogon $Q_{0}$, however, yields $b_{4}=\mathbf{o}_{d}$; we put $\lambda_{4}:=0$ and perform a new case study. The affine shape of $Q_{k}$ tends towards the prototype $T_{3}$. Figure 3 displays $Q_{0}$ and the following generations up to $Q_{6}$.

\section{Conclusion}

We studied affine iterations transforming an initial $n$-gon $Q_{0}$ in $\mathbb{E}^{d}(d>1)$ into successive generations of $n$-gons $Q_{k}$. The Affine Regularization Theorem in this paper does not only extend the results in [5] to dimensions $d>2$; surprisingly, even for dimensions $d>2$ the regularization leads to planar, regular prototypes no matter which generic input $n$-gon $Q_{0}$ we start with. For very specific input $n$-gons $Q_{0}$, though, the same algorithm seems to regularize in a different way. The understanding of this phenomenon completes the results.

\section{Acknowledgements}

Open access funding provided by Graz University of Technology.

Open Access. This article is distributed under the terms of the Creative Commons Attribution 4.0 International License (http://creativecommons.org/licenses/ by/4.0/), which permits unrestricted use, distribution, and reproduction in any medium, provided you give appropriate credit to the original author(s) and the source, provide a link to the Creative Commons license, and indicate if changes were made.

\section{References}

[1] Donisi, S., Martini, H., Vincenzi, G., Vitale, G.: Polygons derived from polygons via iterated constructions. Electron. J. Differ. Geom. Dyn. Syst. 18, 14-31 (2016)

[2] Nicollier, G.: Convolution filters for polygons and the Petr-DouglasNeumann theorem. Contrib. Algebra Geom. 54, 701-708 (2013). doi:10.1007/ s13366-013-0143-9 
[3] Pech, P.: The Harmonic analysis of polygons and Napoleon's theorem. J. Geom. Graph. 5(1), 13-22 (2001)

[4] Radcliffe, D.: Inscribed polygons and the Fourier transform. https://mathblag. wordpress.com/2013/10/08/inscribed-polygons-and-the-fourier-transform/. 26 April 2016.

[5] Röschel, O.: Polygons and iteratively regularizing affine transformations. Contrib. Algebra Geom. (in print). doi:10.1007/s13366-016-0313-7

[6] Schoenberg, I.J.: The finite Fourier series and elemenatry geometry. Am. Math. Mon. 57, 390-404 (1950)

[7] Ziv, B.: Napoleon-like configurations and sequences of triangles. Forum Geom. 2, 115-128 (2002)

Johann Lang, Sybille Mick and Otto Röschel

Institute of Geometry, NAWI Graz

Graz University of Technology

Kopernikusgasse 24

8010 Graz

Austria

e-mail: johann.lang@tugraz.at

Sybille Mick

e-mail: mick@tugraz.at

Otto Röschel

e-mail: roeschel@tugraz.at

Received: October 27, 2016.

Revised: January 27, 2017. 